\title{
Realizing NiO nanocrystals from a simple chemical method
}

\author{
NEELABH SRIVASTAVA and P C SRIVASTAVA* \\ Department of Physics, Banaras Hindu University, Varanasi 221 005, India
}

MS received 25 June 2009; revised 28 August 2010

\begin{abstract}
Nanocrystalline $\mathrm{NiO}$ has been prepared successfully by a simple chemical route using $\mathrm{NiCl}_{2} \cdot 6 \mathrm{H}_{2} \mathrm{O}$ and $\mathrm{NaOH}$ aqueous solution at a temperature of $70^{\circ} \mathrm{C}$. The prepared material has been characterized from XRD, SEM, and $\mathrm{M}-\mathrm{H}$ characteristics. It has been found that $\mathrm{NiO}$ nanocrystals have been formed which shows a superparamagnetic/superantiferromagnetic behaviour.
\end{abstract}

Keywords. Nanostructures; chemical synthesis; X-ray diffraction; SEM.

\section{Introduction}

Nanomaterials have become the caption of the activity now a days in the area of material science. Magnetism of nanomaterials has become a significant concern in nanoscience due to the expected spectacular properties for its wide applications in diverse fields such as high density recording media, spin valves, magnetic resonance imaging, ferrofluid technology and magnetocaloric refrigeration (Gleiter 2000; McHenry and Laughlin 2000). More recently, magnetic properties were experimentally studied in some antiferromagnetic nanoparticles such as $\mathrm{MnO}$ (Kodama et al 1997) and $\alpha-\mathrm{Fe}_{2} \mathrm{O}_{3}$ (Bødker et al 2000a, b). Nanoparticles with antiferromagnetic ordering of spins have received much interest due to their interesting properties such as superparamagnetism, enhanced magnetic moments and field reversal due to quantum tunneling (Zhang et al 1997; Chen and Zhang 1998). In this context, nanosized nickel oxide (NiO) is an interesting material due to its useful electronic (Biju and Khadar 2001) and magnetic properties (Bødker et al 2000a, b).

So far many synthesis routes have been reported for preparing $\mathrm{NiO}$ nanocrystals. For example, Qian and coworkers synthesized $\mathrm{NiO}$ nanoplatelets by thermal decomposition of the prepared nickel hydroxide precursor (Wang et al 2006). Wang and coworkers prepared $\mathrm{NiO}$ nanowires by annealing the freshly prepared precursor (Xu et al 2003). Deki et al (2003) prepared highly concentrated nanosized $\mathrm{NiO}$ by means of vacuum evaporation of $\mathrm{Ni}$ on $\mathrm{NH}_{2}$-terminated $\mathrm{PEO}$ film. Zheng and Zhang (2007) reported a molten-salt synthesis method using $\mathrm{NaCl}$ as a flux at $1000^{\circ} \mathrm{C}$ to prepare $\mathrm{NiO}$ nanoparticles with uniform morphology of hexagon plates. In this paper, we report on the preparation of $\mathrm{NiO}$ nanocrystals by a simple chemical method using aqueous solution of nickel chloride and sodium hydroxide at $70^{\circ} \mathrm{C}$. The formed material has been characterized from XRD, SEM and $\mathrm{M}-\mathrm{H}$ behaviour to show the formation of $\mathrm{NiO}$ nanocrystals.

\footnotetext{
*Author for correspondence (pcsrivastava50@gmail.com)
}

\section{Experimental}

$\mathrm{NiO}$ nanoparticles have been prepared by sol-gel chemical method. The chemicals used in the preparation were of analytical grade and were used without further purification. $0 \cdot 1 \mathrm{M}$ of $\mathrm{NiCl}_{2} \cdot 6 \mathrm{H}_{2} \mathrm{O}$ and $0 \cdot 3 \mathrm{M}$ of $\mathrm{NaOH}$ pellets were dissolved in appropriate amount of distilled water separately. Aqueous solution of $\mathrm{NiCl}_{2} \cdot 6 \mathrm{H}_{2} \mathrm{O}$ was kept on a heated magnetic stirrer (at $70^{\circ} \mathrm{C}$ ) and $\mathrm{NaOH}$ solution was added drop-wise till the turbidity occurred, for $10 \mathrm{~h}$. Thus, the green gel was formed which was washed several times with distilled water. Then, the sample was allowed to dry in an oven (furnace) at $300^{\circ} \mathrm{C}$ for $5 \mathrm{~h}$. Finally, the dried powder was ground in an agate mortar.

To identify the structure and phase purity of the prepared sample, powder X-ray diffraction (XRD) measurement was performed by using a Philips PW-1710 diffractometer with $\mathrm{CuK}_{\alpha}$ radiation $(\lambda=1.540598 \AA)$ at room temperature. The surface morphology of the prepared sample was carried out by scanning electron microscopy (SEM, Philips XL-20). Magnetization measurement $(\mathrm{M}-\mathrm{H}$ characteristics) of the sample was recorded from a vibrating sample magnetometer (VSM) where magnetic field could be varied up to 1.8 Tesla.

\section{Results and discussion}

The X-ray diffraction data (table 1) have been recorded for powdered sample as shown in figure 1. This data clearly shows distinct peaks at $2 \theta$ of $37 \cdot 060,43.095,62.620$, $75 \cdot 090$, and $79 \cdot 185$. The peaks have been identified as peaks of cubic $\mathrm{NiO}$ crystallites with various diffracting planes [111], [200], [220], [311] and [222]. The XRD pattern revealed the formation of cubic phase of $\mathrm{NiO}$ (JCPDS Card 47-1049) with lattice constant, $a=4 \cdot 1771 \AA$. The other peaks observed at an angle $(2 \theta)$ of $31 \cdot 575,56 \cdot 310,66 \cdot 050$ which have been identified as $\mathrm{Ni}_{2} \mathrm{O}_{3}$ corresponds to [002], [202] and [004] crystal planes, respectively. A peak 
Table 1. XRD data of $\mathrm{NiO}$ nanoparticles.

\begin{tabular}{lllclcl}
\hline $\begin{array}{l}\text { Angle } \\
(2 \theta)\end{array}$ & $\begin{array}{l}d \text {-value } \\
(\AA)\end{array}$ & $\begin{array}{l}\text { Peak } \\
\text { width }(2 \theta)\end{array}$ & $\begin{array}{l}\text { Peak intensity } \\
(\text { counts })\end{array}$ & $\begin{array}{l}\text { Relative } \\
\text { intensity (counts) }\end{array}$ & $\begin{array}{l}\text { Identification } \\
\text { (from XRD std. data) }\end{array}$ & $\begin{array}{l}\text { Crystallite } \\
\text { size }(\mathrm{nm})\end{array}$ \\
\hline $37 \cdot 060$ & $2 \cdot 4298$ & $0 \cdot 200$ & 353 & $19 \cdot 7$ & $\mathrm{NiO}[111]$ & $41 \cdot 77$ \\
43.095 & $2 \cdot 1025$ & $0 \cdot 250$ & 590 & $32 \cdot 8$ & $\mathrm{NiO}[200]$ & $34 \cdot 19$ \\
62.620 & 1.4859 & $0 \cdot 350$ & 262 & $14 \cdot 6$ & $\mathrm{NiO}[220]$ & $26 \cdot 60$ \\
75.090 & 1.2672 & $0 \cdot 150$ & 313 & $17 \cdot 4$ & $\mathrm{NiO}[311]$ & $67 \cdot 25$ \\
$79 \cdot 185$ & 1.2062 & $0 \cdot 400$ & 55 & $3 \cdot 0$ & $\mathrm{NiO}[222]$ & $25 \cdot 70$ \\
\hline
\end{tabular}

observed at $2 \theta$ of $45 \cdot 315$ have been identified for $\mathrm{Ni}$ [111] phase. A peak at $2 \theta$ of 27.230 is frequently observed which corresponds to the cello tape, used for holding the samples.

XRD data (table 1) shows the peak width, $2 \theta$, for various peaks. The broadened peak shows the nanometer-sized crystallites. The crystallite size has been estimated (by using the Scherrer relation (Eberhart 1989)), which has been found to vary between $25 \mathrm{~nm}$ and $67 \mathrm{~nm}$ for various identified diffraction peaks (table 1).

The SEM micrographs of the prepared NiO crystals are shown in figure 2. The SEM micrograph shows cubic crystallites. Some of the cubic crystallites are marked by the boxes in figure 2. Moreover, the micrograph also shows the agglomeration of the crystallites. The agglomeration may occur due to the crystallites being of nanodimension. The small nanocrystals possess large surface energy, which leads the nanocrystals to aggregate in order to lower their surface energy during crystal growth.

$\mathrm{M}-\mathrm{H}$ behaviour, for in plane $(\|)$ and out of plane $(\perp)$ applied magnetic fields, of the prepared $\mathrm{NiO}$ nanoparticles is shown in figure 3 . The magnetization has been observed not to saturate up to a maximum applied field of $18 \mathrm{kOe}$. The $\mathrm{M}-\mathrm{H}$ characteristics do not show any significant hysteresis,

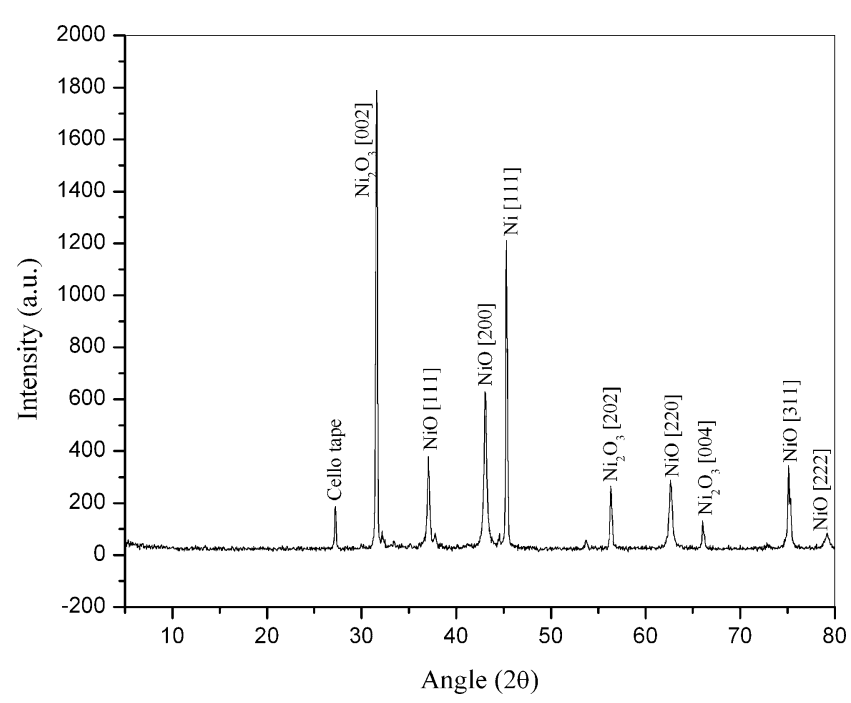

Figure 1. XRD pattern of $\mathrm{NiO}$ nanoparticles. coercivity, and retentivity and thus it clearly shows the feature of superantiferromagnetic/superparamagnetic behaviour.

The magnetic behaviour of the particle surface differs from that of core. $\mathrm{NiO}$ is basically antiferromagnetic below Neel temperature $(523 \mathrm{~K})$. The magnetic ordering in $\mathrm{NiO}$ nanoparticles results from the super exchange interaction (Anderson 1950). This super exchange interaction is an indirect exchange mechanism whereby electrons are shared between $3 d$ orbitals of metal ions, i.e. $\mathrm{Ni}^{+2}$ ions and $2 p$ orbitals of the intervening oxygen ions $\left(\mathrm{O}^{-2}\right.$ ions). This interaction strength is proportional to the overlap between these orbitals; hence it depends on the bond length and angle between the metal ion and oxygen ions. When some exchange bonds are broken from the surface, there can be frustration and spin disorder. As the particle size is reduced to nanometer size, then surface to volume ratio $(\alpha 1 / r$, where $r$ is the particle size) increases and hence the role of the surface atoms in the magnetic behaviour becomes significant and energy consideration favours the formation of single domain particles. This could also show unique magnetic properties such as superparamagnetism (Kittel 1974). Neel (1962) suggested a two-sublattice model, one with spin 'up' and another with spin 'down' at the surface.

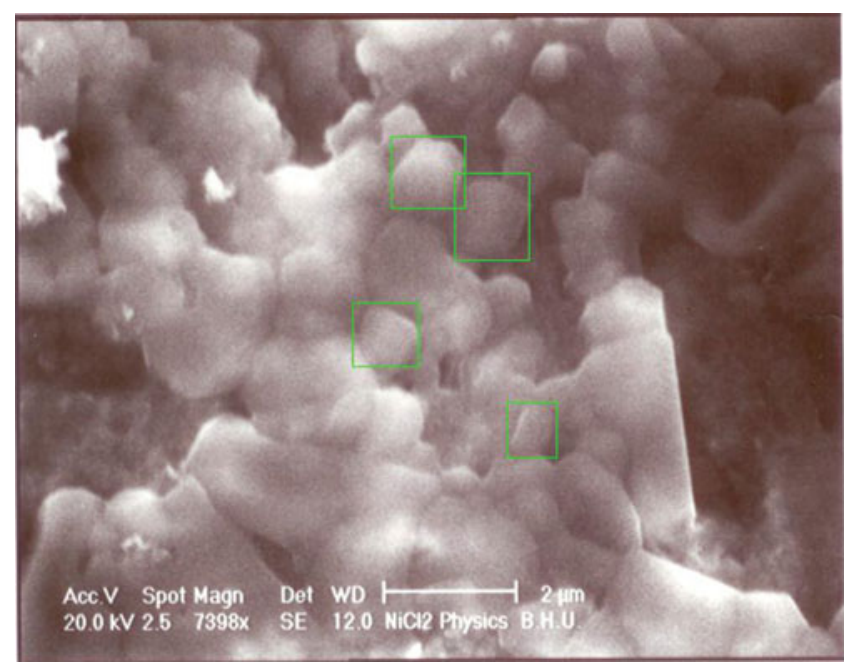

Figure 2. SEM micrograph of $\mathrm{NiO}$ nanoparticles prepared by heating $\mathrm{Ni}(\mathrm{OH})_{2}$ precursor. 


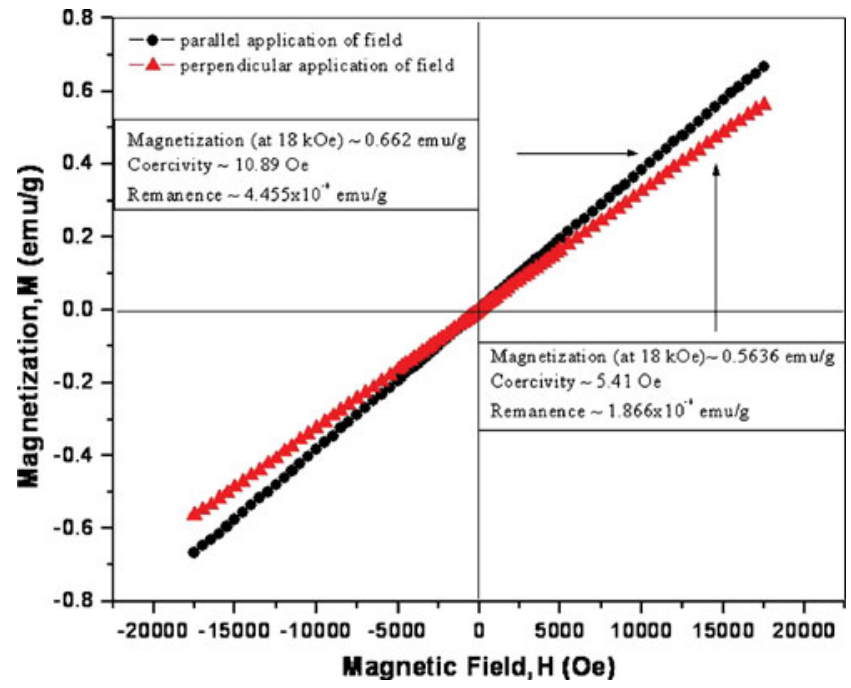

Figure 3. $\mathrm{M}-\mathrm{H}$ characteristics of $\mathrm{NiO}$ nanoparticles.

There is a net magnetic moment in antiferromagnetic nanoparticles due to the non-compensation of surface spins. The paramagnetic susceptibility due to the noncompensation of surface spins can easily dominate over the antiferromagnetic contribution itself. Because of the structural disorder, the surface spin can be more easily deviated from the antiferromagnetic alignment by a magnetic field. Since, a surface spin has smaller number of neighbours than it would have in bulk and hence experiences a reduced mean field. For very small particle size, the surface area is much larger and hence makes a large contribution to the magnetization.

The two main contributions to the magnetization are:

$$
M(H, T)=M_{0}(T)+\chi_{\mathrm{AF}} H(T),
$$

where the first term, $M_{0}(T)$ in the magnetization equation is responsible for the rapid increase in the magnetization due to the non-compensation of surface spins whereas the second term $\chi_{\mathrm{AF}} H(T)$ is responsible for the non-saturation of the magnetization at high fields due to some antiferromagnetic contribution.

From figure 3 , it is clear that the magnetization behaviour of $\mathrm{NiO}$ nanoparticles shows more or less linear dependence on the applied field. Schuele and Deetscreek (1962) had also earlier reported the linear dependence of magnetization on the applied field for $\mathrm{NiO}$ nanoparticles with an average particle size between $21 \AA$ and $\sim 1000 \AA$. From figure 3 , the susceptibility, $\chi$, has been calculated to be $\sim 39 \cdot 6 \times 10^{-6} \mathrm{emu} / \mathrm{g}$ (for in plane magnetization) and $\sim 36 \times 10^{-6} \mathrm{emu} / \mathrm{g}$ (for out of plane magnetization). These values obtained are approximately five times the antiferromagnetic susceptibility of bulk $\mathrm{NiO}$ at room temperature, which is reported as $\sim 8 \times 10^{-6} \mathrm{emu} / \mathrm{g}$ (Singer 1956).

This observation is in good agreement with the statement made by Neel (1961) in which he has already discussed two surface effects: (i) weaker mean field of the surface spins implies that they can be more easily deviated from antiferromagnetic alignment by a magnetic field. This will give rise to an enhanced antiferromagnetic susceptibility, which Neel called 'superantiferromagnetism' and (ii) Neel recognized that the effect of reduced surface magnetization which was observed in Monte Carlo simulations (Wildpaner 1974) for ferromagnets may be even more important for antiferromagnetic particles for which 'superparamagnetism' arises from the uncompensated spins.

Again, it is also in good agreement with the result reported by Khadar et al (2003) that at room temperature $\mathrm{NiO}$ nanoparticles with average particle size $>\sim 10 \mathrm{~nm}$ may exhibit superantiferromagnetism with the magnetization curve showing a linear dependence on the applied field. Hence, the susceptibility values observed are larger in comparison with the antiferromagnetic susceptibility of bulk $\mathrm{NiO}$.

Thus, the above magnetic properties show typical behaviour of $\mathrm{NiO}$ nanoparticles.

\section{Conclusions}

$\mathrm{NiO}$ nanocrystals have been prepared by the thermal decomposition of the as-prepared $\mathrm{Ni}(\mathrm{OH})_{2}$ precursor which is prepared from the aqueous solution of $\mathrm{NiCl}_{2} \cdot 6 \mathrm{H}_{2} \mathrm{O}$ and $\mathrm{NaOH}$ at $70^{\circ} \mathrm{C}$. XRD features have revealed the formation of cubic phase of $\mathrm{NiO}$ nanocrystals. SEM micrograph also shows the features of cubic crystallite and aggregation of nanocrystallites. $\mathrm{M}-\mathrm{H}$ characteristics clearly show the feature of superparamagnetism/ superantiferromagnetism which is a typical behaviour of $\mathrm{NiO}$ nanocrystallites.

\section{References}

Anderson P W 1950 Phys. Rev. 79350

Biju V and Khadar M A 2001 Mater. Sci. Engg. A304-306 814

Bødker F, Hansen M F, Bender Koch C, Lefmann K and Mørup S 2000a Phys. Rev. B61 6826

Bødker F, Hansen M F, Koch C B and Mørup S 2000b J. Magn. Magn. Mater. 22132

Chen Qi and Zhang Z John 1998 Appl. Phys. Lett. 733156

Deki S, Yanagimoto H, Hiraoka S, Akamatsu K and Gotoh K 2003 Chem. Mater. 154916

Eberhart J P 1989 Analyse Structurale et Chimiques des Matériaux (Paris: Ed. Dunod) 46 p. 407

Gleiter H 2000 Acta Mater. 481

Khadar M Abdul, Biju V and Akihisa Inoue 2003 Mater. Res. Bull. 381341

Kittel C 1974 Introduction to solid state physics (New Delhi: Wiley Eastern Private Limited) p. 570

Kodama R H, Makhlouf S A and Berkowitz A E 1997 Phys. Rev. Lett. 791393

McHenry M E and Laughlin D E 2000 Acta Mater. 48223 
Neel L 1961 C. R. Acad. Sci. Paris 252 4075, 253 9, 203, 1286 Neel L 1962 Low temperature physics (eds) C Dewitt et al (New York: Gordon and Breach) p. 413

Schuele W J and Deetscreek V D 1962 J. Appl. Phys. Suppl. 331136 Singer J R 1956 Phys. Rev. 104929

Wang X, Li L, Zhang Y, Wang S, Zhang Z, Fei L and Qian Y 2006 Cryst. Growth \& Des. 62163
Wildpaner V 1974 Zeitschrift fur Physik A Hadrons and Nuclei 270215

Xu C, Hong K, Liu S, Wang G and Zhao X 2003 J. Cryst. Growth 255308

Zhang X X, Tejada J, Hernandez J M and Ziolo R F 1997 Nanostruct. Mater. 9301

Zheng Y and Zhang M 2007 Mater. Lett. 613967 